\title{
The Nano-Imaging Method: A 3D Super-Resolution Technique for Imaging of Filamentous Actin by Directed Motion of Gold Nanoparticles
}

\author{
Laura C. Estrada and Enrico Gratton
}

University of California, Irvine

More than 100 years ago, Ernst Abbe established that diffraction of light prevents optical-based microscopes from having spatial resolution beyond a value comparable to the illumination wavelength. However, since the mid-90s, several super-resolution techniques have emerged. Stimulated Emission Depletion (STED), Saturated Structured-Illumination Microscopy (SSIM), Photo-Activated Localization Microscopy (PALM) and Stochastic Optical Reconstruction Microscopy (STORM) are some of the imaging techniques now available.

In this contribution, I will present a new optical super-resolution method based on a nanoparticle (NP) tracking/pushing principle. Although the image of a single NP is diffraction-limited, the precision of determining its position scales with the inverse of the square root of the number of collected photons. Using the nano-imaging method, we can push single gold NPs along biological fibers by using a two-photon microscope and the localized surface plasmon at the NP. Gold NPs binds to collagen and actin but it can gently slide over the fiber without detaching. While the particle is sliding, we determine its 3D location with nanometer precision by a 3D tracking algorithm. The recorded trace follows the 3D structure of the underlying fiber without the need of staining. The latest examples that illustrate the capabilities of the nano-imaging method will be discussed. 